\title{
Trasplante pulmonar en pacientes portadores de fibrosis pulmonar: caracterización de una cohorte de 87 pacientes
}

\author{
MARÍA TERESA PARADA C.*, JOEL MELO T.*, CLAUDIA SEPÚLVEDA L.**, DAVID LAZO P.*, \\ VIRGINIA LINACRE S.**, ELI VILLALABEITÍA R.*, JOSÉ MIGUEL CLAVERO R.*, \\ MACARENA LAGOS C.*, CRISTINA HERZOG O.*, LORENA CALABRÁN R.*, PATRICIO VALLEJO V.**, \\ ERIKA DONOSO G.**, FRANCESCA GAJARDO Z.** y MAURICIO SALINAS F.**
}

\section{Lung transplantation in 87 patients with idiopathic pulmonary fibrosis}

Pulmonary fibrosis is a progressive disease. Lung transplantation is the only effective therapy for a group of patients. Objective: To evaluate results of lung transplantation for fibrosis up to a 5 years of follow up. Methodology: Retrospective review of clinical records of patients subjected to lung transplantation from Clínica Las Condes and Instituto Nacional del Tórax. Demographic data, type of transplant, baseline and post-transplant spirometry and 6 min walked distance (6MWT), early and late complications and long-term survival rate were analyzed. Results: From 1999 to 2015, 87 patients with pulmonary fibrosis were transplanted, in average they were 56 years old, $56 \%$ were male, $89 \%$ of patients were subjected to a single lung transplant. $16 \%$ of them were in urgency. Baseline and 1-3-5 years for FVC were 49-73-83 and 78\% of the reference values and for 6MWT were 280, 485, 531 and 468 meters respectively. Predominant complications $<1$ year post-transplant were: acute rejection $(30 \%)$ and infections (42\%). Complications after 1 year of transplantation were chronic graft dysfunction (DCI) $57 \%$ and neoplasms (15\%). The main causes of mortality $>1$ year were DCI (45\%) and neoplasms (11\%). The estimated 1, 3 and 5 year survival rate were 84, 71 and 58\% respectively. Conclusions: Lung transplantation in patients with pulmonary fibrosis improves their quality of life and survival rate. The monopulmonary technique is efficient in the long term. Acute rejection and infection were the most common early complications and chronic graft dysfunction was the prevalent long-term complication.

Key words: Lung transplantation, pulmonary fibrosis.

\section{Resumen}

La Fibrosis pulmonar es una enfermedad progresiva y el trasplante constituye una terapia efectiva para un grupo de pacientes. Objetivo: Evaluar los resultados del trasplante pulmonar por fibrosis a 5 años. Metotodología: Revisión retrospectiva de registros de trasplante pulmonar de la Clínica Las Condes e Instituto Nacional del Tórax. Se analizaron datos demográficos, tipo de trasplante, función pulmonar basal y post-trasplante, complicaciones precoces y tardías y sobrevida a largo plazo. Resultados: Entre 1999 y 2015 ambos centros trasplantaron 87 pacientes por fibrosis pulmonar. Los pacientes tenían una edad promedio de 56 años, 56\% eran del género masculino y se usó técnica monopulmonar en $89 \%$ de ellos. $16 \%$ de los pacientes se encontraba en urgencia. Los resultados espirométricos y la distancia caminada en 6 minutos (T6 min) basales y a 1- 3 - 5 años fueron: CVF 49-73- 83 y 78\% del valor teórico y T6 min fue 280, 485, 531 y 468 metros respectivamente. Complicaciones predominantes $<1$ año fueron: rechazo agudo 30\% e infecciones $42 \%$. Complicaciones $>1$ año fueron: disfunción crónica del injerto (DCI) 57\% y neoplasias 15\%. Las causas de mortalidad > 1 año fueron DCI 45\% y neoplasias 11\%. La sobrevida estimada a uno, 3 y 5 años fue 84, 71 y 58\% respectivamente. Conclusiones: El trasplante en pacientes con fibrosis pulmonar, permite mejorar la calidad de vida y sobrevida de estos pacientes. La técnica monopulmonar es eficiente a largo plazo. En las complicaciones precoces predominaron el rechazo agudo e infecciones y a largo plazo la DCI.

Palabras clave: Trasplante pulmonar, fibrosis pulmonar.

\footnotetext{
* Centro de Trasplantes Clínica Las Condes. Las Condes, RM, Chile.

** Instituto Nacional del Tórax, Providencia, RM, Chile.
} 


\section{Introducción}

La fibrosis pulmonar idiopática (FPI) es la forma más frecuente de las neumonías intersticiales de origen desconocido, siendo su característica histopatológica y radiológica habitual la de un patrón de neumonía intersticial usual (UIP). La historia natural es variable, asociándose a un deterioro progresivo de la función pulmonar. $\mathrm{La}$ sobrevida media de los pacientes es de 3 años posterior al diagnóstico y 30 a $40 \%$ a los 5 años de realizado el trasplante ${ }^{1}$.

A pesar de los avances en investigación clínica, la fibrosis pulmonar permanece como una enfermedad incurable y cuyas opciones terapéuticas son limitadas. En la actualidad sólo la pirfenidona y nuevos estudios con nintedanib han mostrado enlentecer la progresión del daño pulmonar ${ }^{2}$.

El trasplante pulmonar es en la actualidad la única terapia que ha probado beneficios clínicos determinados por corrección de la insuficiencia respiratoria basal, mejorando la calidad de vida y extendiendo la sobrevida para un grupo seleccionado de pacientes ${ }^{3}$. La escasez de donantes es la limitación primaria para la realización de trasplantes por lo que los criterios de selección de candidatos son estrictos, requiriéndose identificar adecuadamente la forma de progresión de la enfermedad, para disminuir el riesgo de fallecer durante la permanencia en lista de espera. En Estados Unidos desde el año 2005 se aplica un criterio de puntuación de gravedad denominado LAS (Lung Allocation Score), el cual ha permitido priorizar el trasplante en pacientes portadores de Fibrosis pulmonar. Las variables de peor pronóstico en este puntaje son el rápido deterioro de los valores espirométricos, el aumento sostenido de requerimientos de oxígeno, la aparición de hipercapnia, el desarrollo de hipertensión pulmonar progresiva y la necesidad de ventilación mecánica no invasiva (VMNI). Esta patología ha desplazado a la EPOC como primera indicación de trasplante en los diferentes centros de trasplante pulmonar a nivel mundial ${ }^{4}$. En nuestro país desde el año 2012 se han establecido criterios de priorización diferentes al LAS, relacionados al uso de ventilación mecánica no invasiva, requerimiento de altas concentraciones de oxígeno o necesidad de soporte extracorpóreo que determinan la urgencia del trasplante a nivel nacional, permitiendo así mejorar la sobrevida de un grupo de pacientes.

La técnica quirúrgica puede ser mono o bipulmonar, siendo esta última la que muestra mejores resultados funcionales a largo plazo, aunque es de elección principalmente para pacientes más jóvenes $^{5}$. En nuestro país predomina la técnica de trasplante monopulmonar.

El objetivo de esta publicación es comunicar los resultados funcionales, complicaciones y sobrevida a largo plazo obtenidos a partir de la cohorte nacional con mayor número de pacientes trasplantados por fibrosis pulmonar.

\section{Metodología}

Se realizó un análisis retrospectivo de los registros de pacientes trasplantados por fibrosis pulmonar intervenidos en Clínica Las Condes e Instituto Nacional del Tórax entre los años 1999 y 2015. Se obtuvieron antecedentes demográficos, de función pulmonar basal: espirometría, capacidad de difusión de $\mathrm{CO}$ y distancia caminada en 6 min. En ambos centros el protocolo de tratamiento inmunosupresor considera un esquema de triterapia: esteroides, inhibidores de calcineurina (ciclosporina o tacrolimus) y azatioprina o micofenolato mofetil, se utilizó terapia de inducción inmunosupresora con Basiliximab. Considerando además el uso de similar esquema de profilaxis infecciosa. El diagnóstico de rechazo agudo se realizó mediante fibrobroncoscopía con análisis histopatológico de biopsia transbronquial siguiendo la clasificación internacional ${ }^{6}$.

Se consignó el tipo de técnica quirúrgica utilizada, necesidad de soporte con ECMO (oxigenación por membrana extracorpórea) en el perioperatorio y los resultados funcionales respiratorios mediante la realización de espirometría y caminata de 6 min al primer año, a los tres y a los cinco años. Se consideró en su calidad de vida: la suspensión de oxígeno, la independencia en actividades diarias y el retorno a su actividad de base, física y/o laboral previo al desarrollo de insuficiencia respiratoria asociada a fibrosis pulmonar. Se consignó el tipo de complicaciones presentadas durante el primer año y los posteriores. Se elaboraron curvas de sobrevida a uno, 3 y 5 años, mediante el método de Kaplan Meier, registrando las causas de mortalidad precoces y tardías observadas durante el seguimiento. Para el análisis estadístico de los resultados se utilizó el test " $t$ " de Student para datos pareados, considerando significativo un valor de $p<0,05$. Los análisis estadísticos fueron realizados en el software Stata 11.0.

Aspectos éticos: el protocolo de este estudio fue analizado y aprobado por el Comité de Ética de la Clínica Las Condes y del Instituto Nacional del Tórax. 


\section{Resultados}

Entre abril de 1999 y agosto de 2015 en ambos centros se trasplantaron 155 pacientes, $87 \mathrm{de}$ los cuales tenían el diagnóstico confirmado de fibrosis pulmonar. La edad promedio de los pacientes fue 56 años (rango 24 a 70 años), siendo el $56 \%$ de ellos de sexo masculino. La técnica quirúrgica utilizada fue monopulmonar en el $89 \%$ de los trasplantes (Tabla 1). Los resultados de las pruebas funcionales basales al momento de inscripción en la lista de espera mostraron predominio de una limitación ventilatoria restrictiva severa, con CVF promedio de $49 \%$ (rango 20 a $69 \%$ ), Capacidad de difusión de CO promedio de $27 \%$ (rango 16 a $40 \%$ ) y distancia promedio caminada en 6 min: $280 \mathrm{~m}$ (rango 80 a $400 \mathrm{~m}$ ). Todos los pacientes presentaron deterioro funcional progresivo mientras se encontraban en la lista de espera, lo cual impidió un mayor seguimiento de su función respiratoria. Sus requerimientos de oxígeno fueron en ascenso, determinando que el $16 \%$ ingresaran a lista nacional de urgencia por necesitar ventilación mecánica no invasiva. El uso de ECMO post-operatorio fue necesario en $9 \%$ de los casos. Todos los pacientes dados de alta posterior al trasplante habían suspendido el uso de oxígeno. La evolución de la función
Tabla 1. Características demográficas de pacientes trasplantados pulmonares por fibrosis

$\begin{array}{ll}\text { Total pacientes trasplantados (n) } & 155 \\ \text { Fibrosis pulmonar } \mathrm{n}(\%) & 87(56 \%) \\ \text { Hombres (\%) } & 56 \\ \text { Mujeres (\%) } & 44 \\ \text { Edad (años) } & 56 \text { (rango 24-70) } \\ \text { *CVF (promedio) } & 49 \\ \text { *DLCO (promedio) } & 27 \\ \begin{array}{l}\text { Distancia promedio recorrida } \\ \text { en 6 min (m) }\end{array} & 280 \\ \text { Tipo de trasplante: } & \\ \text { Monopulmonar (\%) } & 89 \\ \text { Urgencia (\%) } & 16\end{array}$

*CVF: capacidad vital forzada. DLCO: capacidad de difusión de monóxido de carbono. Ambos índices están expresados en \% de su valor teórico normal.

pulmonar se describe en las Figuras 1 y 2 en las que se evidencia una progresiva mejoría de índices espirométricos, con aumento significativo al primer año post-trasplante $(\mathrm{p}=0,001)$ y en la distancia caminada en 6 min, que se mantiene a los 5 años, con la excepción de los pacientes que presentaron disfunción del injerto. En relación a su limitación física y el retorno a su actividad
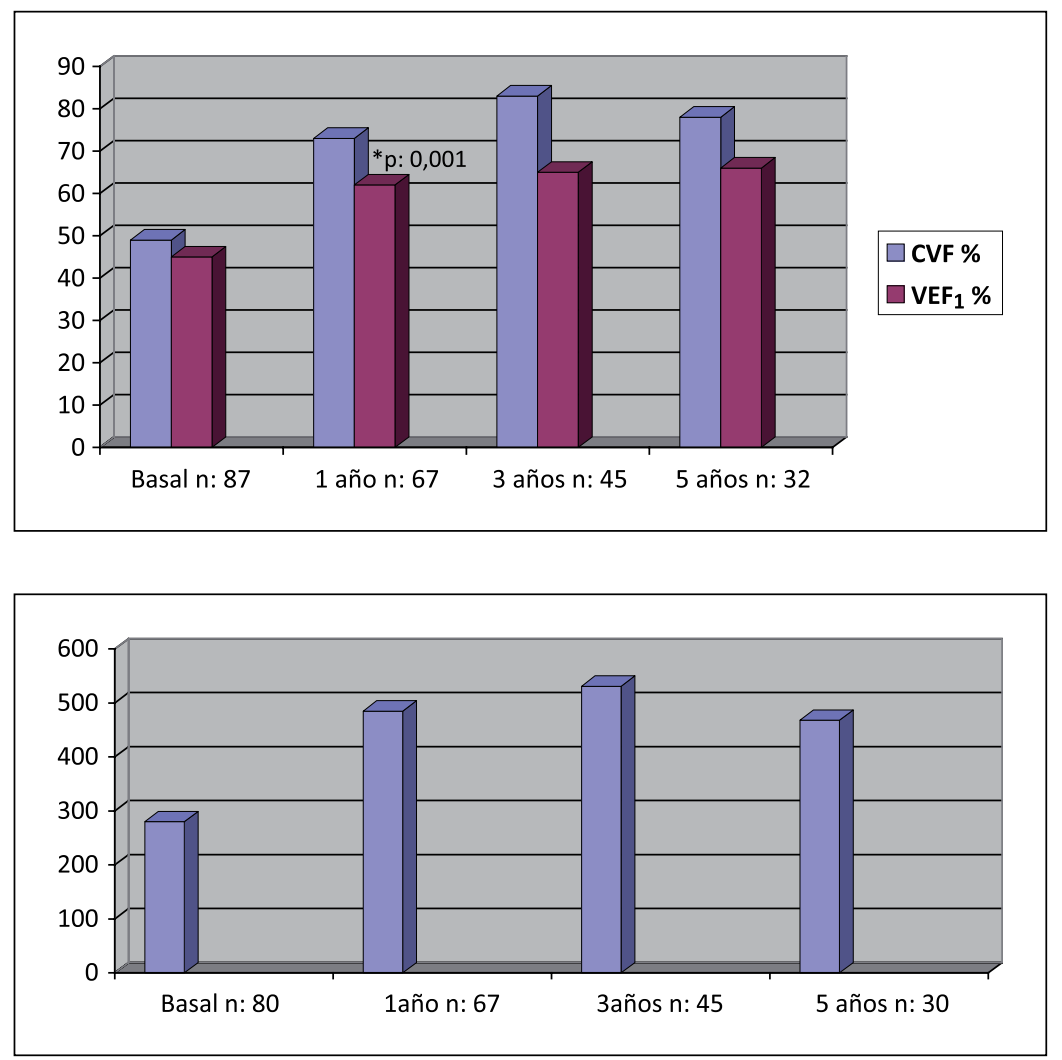

Figura 1. Valores espirométricos en pacientes trasplantados pulmonares. ${ }^{*} \mathrm{VEF}_{1}$ aumentó significativamente un año después del trasplante respecto a su valor basal. Cada par de columnas representa el valor promedio de CVF y $\mathrm{VEF}_{1}$ expresado en $\%$ de su valor teórico.

Figura 2. Distancia recorrida en caminata de 6 min en trasplantados pulmonares. Las columnas representan el promedio de metros recorridos por los pacientes. 

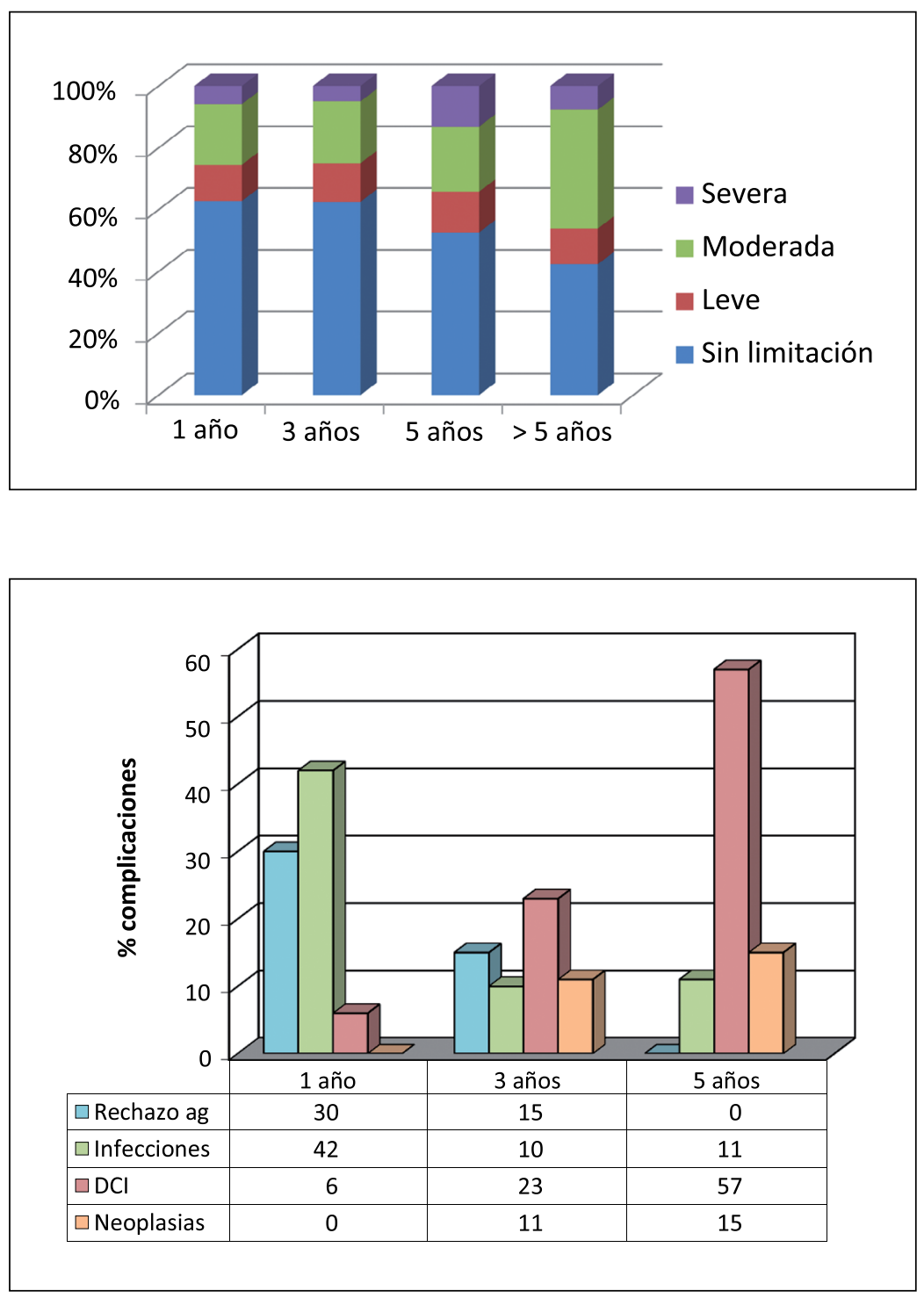

Figura 3. Graduación de la limitación de la actividad física posterior al trasplante en pacientes con fibrosis pulmonar.
Figura 4. Evolución de las complicaciones del trasplante en pacientes con fibrosis pulmonar.

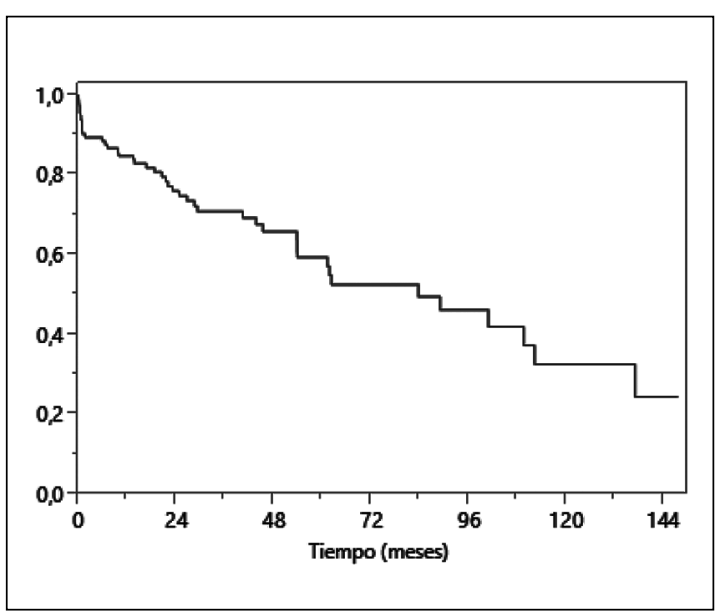

Figura 5. Curva de sobrevida de 87 pacientes trasplantados pulmonares por fibrosis pulmonar (Kaplan Meier). habitual previo al desarrollo de insuficiencia respiratoria por fibrosis, se observó que el $76 \%$ de los pacientes recuperó su capacidad física en el primer año posterior al trasplante y se mantuvo con limitación leve un 52\% a 5 años (Figura 3)

La evolución de las complicaciones en el tiempo se describe en la Figura 4. En el primer año destaca el rechazo agudo en el $30 \%$ de los casos. Las infecciones alcanzaron un total de $42 \%$, siendo de origen bacteriano en un $24 \%$, con predominio de gérmenes Gram negativos del tipo Pseudomonas sp, Klebsiella pneumoniae y Enterobacter $s p$ asociados a casos de bronquitis purulenta, registrándose 7 pacientes con neumonía en este período. La infección aspergilar ocurrió en el $7 \%$ de los casos y en tres pacientes estuvo asociado a lesión de la vía aérea, cuya biopsia mostró 
aspergilosis bronquial invasiva. La enfermedad por citomegalovirus se presentó en el $6 \%$.

Posterior al primer año se observa una notable disminución de complicaciones, apareciendo tardíamente la disfunción crónica del injerto (DCI) $23 \%$ a 3 años y $57 \%$ a 5 años. También en forma tardía se detectan neoplasias $(15 \%)$, y se reduce la incidencia de infecciones a un 11\%. Además del desarrollo de neoplasias cutáneas cabe destacar que posterior a los 3 años se encontraron 5 adenocarcinomas (dos pulmonares, uno gástrico, uno vesical y uno de próstata) y 3 neoplasias de tipo hematológico. Las principales causas de mortalidad posterior al primer año de trasplante fueron DCI (45\%), neoplasias (14\%) e infecciones (9\%). La sobrevida estimada de esta cohorte fue de $84 \%$ dentro del primer año, $71 \%$ al tercer año y $58 \%$ a los 5 años de realizado el trasplante.

\section{Discusión}

El trasplante pulmonar es aceptado como una opción terapéutica para diversas patologías respiratorias en etapa avanzada y refractaria al tratamiento médico, en donde la fibrosis pulmonar constituye la principal indicación en la actualidad ${ }^{7}$. Esta enfermedad se caracteriza por la progresión de síntomas respiratorios como tos, disnea, el desarrollo de insuficiencia respiratoria que determina una limitación progresiva de la actividad física diaria e importante deterioro de la calidad de vida, llevando finalmente a la muerte del paciente.

El objetivo del injerto pulmonar es mejorar la sobrevida y la calidad de vida, y ante la escasez de donantes es importante que los recursos disponibles sean optimizados en cuanto a su uso. Debido a la falta de cura definitiva para la fibrosis pulmonar, existen guías establecidas para la adecuada selección de candidatos para ser sometidos a trasplante ${ }^{8}$, las que sugieren derivar los pacientes en etapa precoz, ya que se ha demostrado que es en este grupo de pacientes donde se concentra la mayor mortalidad en la lista de espera ${ }^{9}$.

En nuestro país y en los registros internacionales, la fibrosis pulmonar constituye la principal indicación de trasplante pulmonar. De la cohorte analizada en esta comunicación la fibrosis pulmonar alcanza un $56 \%$ y es el diagnóstico predominante en la lista de espera nacional. Este grupo tiende a presentar un deterioro clínico y funcional más acelerado que otras patologías, lo que llevó a implementar en Estados Unidos el puntaje de gravedad LAS, el cual favorece a estos pacientes ${ }^{10}$. En nuestro país desde el año 2012 se considera como causal de ingreso a lista de urgencia a aquellos pacientes que declinan aceleradamente su función pulmonar requiriendo altas concentraciones de oxígeno o ventilación mecánica no invasiva. En nuestra cohorte de los 18 pacientes en urgencia nacional 13 son portadores de fibrosis pulmonar.

La técnica quirúrgica constituye un tema en revisión con un aumento de los trasplantes bipulmonares en Estados Unidos por la asociación de un mejor pronóstico funcional a largo plazo, pero en contraposición con la escasez de donantes ${ }^{11}$. En nuestro país existe un predominio de técnica monopulmonar para este tipo de pacientes, alcanzando al 89\% de los casos analizados en esta comunicación. Esta técnica ha mostrado resultados funcionales muy favorables a largo plazo, como lo demuestra nuestro seguimiento espirométrico y la distancia caminada en 6 min 5 años post-trasplante.

El uso de inmunosupresión asociado a la conexión de la vía aérea al ambiente favorece las complicaciones de tipo infeccioso, principalmente las de origen bacteriano durante los primeros meses post-trasplante, las que alcanzan en algunas series hasta $50 \%{ }^{12}$. En nuestra serie se observó un $42 \%$ de este tipo de complicaciones durante el primer año, cifra que disminuye progresivamente posterior a la cicatrización de la sutura bronquial y disminución de la inmunosupresión. El uso de profilaxis infecciosa universal ha disminuido el desarrollo de gérmenes oportunistas de tipo $\mathrm{No}$ cardia, Pneumocystis jiroveci y Aspergillus sp. La otra frecuente complicación es el desarrollo de al menos un episodio de rechazo agudo el que se presenta con una frecuencia de 30 a $50 \%{ }^{13}$. En nuestra serie el rechazo agudo alcanzó un 30\% durante el primer año.

El desarrollo de disfunción crónica de injerto (DCI), manifestada principalmente como síndrome de bronquiolitis obliterante, constituye la principal limitación del trasplante a largo plazo, alcanzando alrededor del $50 \%$ a los 5 años ${ }^{13-14}$. En nuestro seguimiento alcanza un $23 \%$ a 3 años y $57 \%$ a los 5 años.

La aparición tardía de neoplasias se describe con una frecuencia cercana al 13\% de los trasplantados por fibrosis a 5 años ${ }^{13-15}$ : en nuestra serie la frecuencia llegó a un $15 \%$ a 5 años. En relación a las causas de mortalidad a largo plazo observamos en esta cohorte que la principal es la DCI (45\%) seguida de neoplasias (14\%), siendo infrecuentes las infecciones como causa de mortalidad después de primer año post-trasplante $(9 \%)$.

La suspensión del uso de oxígeno asociado a la mejoría progresiva de la función pulmonar permite a los pacientes disminuir su limitación física y dependencia para las actividades de la vida 
diaria, lo que mejora su calidad de vida posterior al trasplante.

La sobrevida del injerto pulmonar ha mejorado en la última década, lo que se atribuye a la disponibilidad de un mejor soporte peri-operatorio incluyendo las técnicas de oxigenación extracorpórea, la optimización de terapia inmunosupresora y la disminución de complicaciones precoces mediante el uso de protocolos de profilaxis infecciosa lo que ha permitido en esta serie una sobrevida estimada a 1, 3 y 5 años de 84, 71 y $58 \%$. Estos porcentajes de sobrevida superan ampliamente a la sobrevida observada con la enfermedad de base.

Entre las limitaciones de este estudio se deben considerar la falta de análisis de comorbilidades que puedan influenciar en los resultados a largo plazo como el tabaquismo y el reflujo gastroesofágico.

\section{Conclusiones}

En un grupo de pacientes portadores de fibrosis pulmonar el trasplante ofrece un tratamiento efectivo a largo plazo que mejora la calidad de vida evidenciado por suspensión de oxígeno y mejoría progresiva de la actividad de vida diaria. El trasplante pulmonar permite una sobrevida mayor que la enfermedad de base. Ante la escasez de donantes, la técnica monopulmonar obtiene resultados funcionales eficientes a largo plazo. Las complicaciones más frecuentes presentadas en el primer año son de tipo infecciosas y la presencia de rechazo agudo y en el largo plazo ha predominado la disfunción crónica de injerto.

\section{Bibliografía}

1.- RAGHU G, COLLARD H R, EGAN J J, MARTÍNEZ F J, BEHR J, BROWN K K, et al. ATS/ERS/JRS/ ALAT Committee on Idiopathic Pulmonary Fibrosis: An Official ATS/ERS/JRS/ALAT Statement: Idiopathic pulmonary fibrosis: Evidence-based guidelines for diagnosis and management. Am J Respir Crit Care Med 2011; 183: 788-824.

2.- KING T E, BRADFORD W Z, CASTRO-BERNARDINI S, FAGAN E A, GLASPOLE I, GLASSBERG M $\mathrm{K}$, et al. A phase 3 trial of pirfenidone in patients with idiopathic pulmonary fibrosis. N Engl J Med 2014; 37 : 2083-92.

3.- KISTLER K D, NALYSNYK L, ROTELLA P, ESSER D. Lung transplantation in idiopathic pulmonary fibrosis: a systematic review of the literature. BMC Pulm Med 2014; 14: 139.doi:10.186/1471-2466-14-139. Review.

4.- EGAN T M, MURRAY S, BUSTAMI R T, SHEARON
T H, MCCULLOUGH K P, EDWARDS L B, et al. Development of the New Lung Allocation System in the United States. Am J Transplant 2006; 6 (5p2): 1212-7.

5.- FORCE S D, KILGO P, NEUJAHR D C, PELAEZ A, PICKENS A, FERNÁNDEZ F G, et al. Bilateral lung transplantation offers better long-term survival, compared with single-lung transplantation, for younger patients with idiopathic pulmonary fibrosis. Ann Thorac Surg 2011; 91: 244-9.

6.- MARTINU T, DONG-FENG C, PALMER S. Acute Rejection and Humoral Sensitization in Lung Transplant Recipients. Proc Am Thorac 2009; 6: 54-65

7.- REGISTRY OF TRANSPLANT RECIPIENTS (SRTR): OPTN/SRTR 2011 Annual Data Report. Rockville, MD: Department of Health and Human Services, Health Resources and Services Administration, Healthcare Systems Bureau, Division of Transplantation; 2012.

8.- WEILL D, BENDEN C, CORRIS P A, DARK J H, DAVIS R D, KESHAVJEE S, et al. A consensus document for the selection of lung transplant candidates. J Heart Lung Transplant 2015; 34: 1-15.

9.- NATHAM S D, SHLOBIN O A, AHMAD S, BURTON N A, BARNETT S D, EDWARDS E. Comparison of wait times and mortality for idiopatic pulmonary fibrosis patients listed for single or bilateral lung transplantation. J Heart Lung Transplant 2010; 29: 1165-71.

10.- MERLO C A, WEISS E S, ORENS J B, BORJA M C, DIENER-WEST M, CONTE J V, et al. Impact of U.S. Lung Allocation Score on survival after lung transplantation. J Heart Lung Transplant 2009; 28: 769-75.

11.- DE OLIVEIRA N C, OSAKI S, MALONEY J, CORNWELL R D, MEYER K C. Lung transplant for interstitial lung disease: outcomes for single versus bilateral lung transplantation. Interact Cardiovasc Thorac Surg 2012; 14: 263-7.

12.- TRIBBLE C G, KRON I L, JONES D R. Lung transplantation in patients 60 years and older: results, complications, and outcomes. Ann Thorac Surg 2006; 82: 1835-41.

13.- CHRISTIE J D, EDWARDS L B, AURORA P, DOBBELS F, KIRK R, RHAMEL A O, et al. The Registry of the International Society for Heart and Lung Transplantation. Report 2009. J Heart Lung Transplant 2009; 28: 1031-49.

14.- PARADA M T, ALBA A, SEPÚLVEDA C. Bronchiolitis obliterans syndrome development in lung transplant patients. Transplant Proc 2010; 42: 331-2.

15.- PARADA M T, SEPÚLVEDA C, ALBA A, SALAS A. Malignancy development in lung transplant patients. Transplant Proc 2011; 17: 363- 8.

Correspondencia a:

Dra. María Teresa Parada C.

Centro de Trasplantes, Clínica Las Condes.

Lo Fontecilla 441, Las Condes, RM. Chile.

Email:mtparada@clc.cl 